\title{
Communication of inwardly projecting neovessels with the lumen contributes to symptomatic intraplaque hemorrhage in carotid artery stenosis
}

\author{
Nobutaka Horie, MD, PhD, ${ }^{1}$ Yoichi Morofuji, MD, PhD, ${ }^{1}$ Minoru Morikawa, MD, PhD, ${ }^{2}$ \\ Yohei Tateishi, MD, PhD, ${ }^{3}$ Tsuyoshi Izumo, MD, PhD, ${ }^{1}$ Kentaro Hayashi, MD, PhD, ${ }^{1}$ \\ Akira Tsujino, MD, PhD, ${ }^{3}$ and Izumi Nagata, MD, $\mathrm{PhD}^{1}$ \\ Departments of ${ }^{1}$ Neurosurgery, ${ }^{2}$ Radiology, and ${ }^{3}$ Neurology and Strokology, Nagasaki University School of Medicine, \\ Nagasaki, Japan
}

OBJECT Recent studies have demonstrated that plaque morphology can contribute to identification of patients at high risk of carotid artery atherosclerosis as well as the degree of stenosis in those with carotid atherosclerosis. Neovascularization of carotid plaques is associated with plaque vulnerability. However, the mechanism of neovascularization in intraplaque hemorrhage (IPH) and its clinical contribution remain undetermined. In this study, the authors aimed to clarify the characteristics of neovessel appearance with a focus on inwardly projecting neovessels, which are reportedly important in plaque advancement.

METHODS Consecutive patients with moderate to severe carotid atherosclerosis who underwent carotid endarterectomy were prospectively analyzed from 2010 to 2014. The neovessel appearance was categorized into 3 groups based on intraoperative indocyanine green (ICG) videoangiography: early appearance of neovessels from the endothelium $(\mathrm{NVe})$, late appearance of neovessels from the vasa vasorum (NVv), and no appearance of vessels. Each neovessel pattern was evaluated with respect to clinical, radiological, and pathological findings including IPH, neovascularization, hemosiderin spots, and inflammation.

RESULTS Of 57 patients, 13 exhibited NVe, 33 exhibited NVv, and 11 exhibited no neovessels. Overall, the interobserver and intraobserver reproducibilities of neovessel appearance were substantial for ICG videoangiography $(\kappa=0.76)$ and at 7 days postoperatively $(\kappa=0.76)$. There were no significant differences in baseline characteristics among the 3 groups, with the exception of a higher percentage of symptomatic presentations in patients with NVe (artery-to-artery embolic infarction in $61.5 \%$ and transient ischemic attack in $23.1 \%$ ). Moreover, patients with NVe exhibited larger infarctions than did those with NVv $\left(9675.0 \pm 5601.9 \mathrm{~mm}^{3} \mathrm{vs} 2306.6 \pm 856.9 \mathrm{~mm}^{3}\right.$, respectively; $\left.p=0.04\right)$. Pathologically, patients with NVe had more severe IPH $\left(47.2 \pm 8.3 \mathrm{~mm}^{2}\right.$ vs $19.8 \pm 5.2 \mathrm{~mm}^{2}$, respectively; $\left.p<0.01\right)$, hemosiderin spots $\left(0.5 \pm 0.2 \mathrm{~mm}^{2}\right.$ vs $0.2 \pm 0.1 \mathrm{~mm}^{2}$, respectively; $\left.p=0.04\right)$, neovessels $\left(0.4 \pm 0.7 \mathrm{~mm}^{2}\right.$ vs $0.1 \pm 0.4 \mathrm{~mm}^{2}$, respectively; $p=$ $0.11)$, and inflammation $\left(1.0 \pm 1.1 \mathrm{~mm}^{2} v \mathbf{v s} 0.6 \pm 0.9 \mathrm{~mm}^{2}\right.$, respectively; $\left.p=0.26\right)$ around the endothelium than did patients with $\mathrm{NVv}$, and all of these parameters were correlated with hyperintensity on time-of-flight MRI. However, the neovessel and inflammation differences were nonsignificant. Interestingly, inflammation was significantly correlated with neovessel formation ( $r=0.43, p=0.0008)$, hemosiderin spots $(r=0.62, p<0.0001)$, and IPH $(r=0.349, p=0.0097)$, suggesting that inflammation may be a key factor in plaque vulnerability.

CONCLUSIONS Communication of inwardly projecting neovessels with the lumen and inflammation synergistically contribute to IPH and symptomatic presentations in patients with carotid stenosis and are more specific than the vasa vasorum. This condition could be a new therapeutic target, and regression of luminal neovessel sprouting and inflammation may help to prevent IPH development and a symptomatic presentation.

http://thejns.org/doi/abs/10.3171/2014.12.JNS142371

KEY WORDS carotid plaque; intraplaque hemorrhage; neovascularization; inflammation; vascular disorders

ABBREVIATIONS CEA = carotid endarterectomy; ICG = indocyanine green; IPH = intraplaque hemorrhage; NASCET = North American Symptomatic Carotid Endarterectomy Trial; NVe = early appearance of neovessels from the endothelium; NVv = late appearance of neovessels from the vasa vasorum; TOF $=$ time of flight.

SUBMITTED October 16, 2014. ACCEPTED December 9, 2014.

INCLUDE WHEN CITING Published online June 19, 2015; DOI: 10.3171/2014.12.JNS142371.

DISCLOSURE This work was supported in part by a Grant-in-Aid for Scientific Research from the Japan Society for the Promotion of Science to Dr. Nagata (no.

21591847) 
$\mathrm{R}$ ECENT studies have focused on plaque vulnerability as well as the degree of stenosis in patients with atherosclerotic carotid artery stenosis. ${ }^{2,3}$ Neovascularization has been proposed as an important factor in plaque vulnerability based on pathological analysis ${ }^{25}$ and experimental animal models,,${ }^{17}$ but in vivo evaluation of the degree of neovascularization within plaques has been difficult. Alternatively, some reports have evaluated contrast enhancement of plaques with ultrasonography, MRI, and CT angiography. ${ }^{7-12,16}$ Therefore, the dynamics of neovascularization and its clinical contribution to the development of intraplaque hemorrhage (IPH) and symptomatic presentation remain unclear. The location of neovessels has also been discussed with respect to whether the vessels are vasa vasorum derived or luminal (endothelial) derived..$^{13,15,18}$

In this study, we first applied indocyanine green (ICG) videoangiography for the dynamic assessment of neovascularization of plaques and aimed to: 1) determine whether the ICG videoangiography-based neovessel appearance exhibits specific characteristics associated with the clinical presentation, and 2) assess the hypothesis that inwardly projecting (luminal) neovascularization is clinically associated with a high risk of IPH, assuming an advanced stage of atherosclerosis.

\section{Methods \\ Study Population}

From April 2010 to April 2014, consecutive patients with more than $50 \%$ carotid stenosis on multidetector CT angiography (according to the North American Symptomatic Carotid Endarterectomy Trial [NASCET] $)^{19}$ who underwent carotid endarterectomy (CEA) were prospectively analyzed in this study. The patients were defined as symptomatic because they had experienced an anterior circulation stroke or transient ischemic attack within 2 weeks of radiological analysis. The diagnosis of stroke was based on the clinical presentation at admission and positive findings on diffusion-weighted MRI. The patients were defined as asymptomatic because they had no clinical symptoms and were found to have more than $80 \%$ carotid stenosis during screening for atherosclerosis. All patients underwent CEA based on the NASCET and American Heart Association/ American Stroke Association guidelines, ${ }^{5,11}$ and surgical specimens were histologically analyzed for all patients. This study was approved by our institutional review board, and all patients gave written informed consent. CEA was performed in all symptomatic patients within 2 months after symptom onset.

\section{Radiological Analysis of Plaques and Infarction}

In all patients, carotid atherosclerotic plaques were imaged with a 3.0-T MRI machine (Signa, GE Medical Systems) using a phased-array coil with a diameter of 3 inches. Magnetic resonance imaging examinations were performed within 2 weeks of the surgical procedure. After an initial imaging survey to determine the position of the carotid plaque, the following MRI sequences were used: 1) for T1-weighted imaging, 2D fast spin echo, TR/
TE 800/11 msec, and echo train length 4; and 2) for 2D time of flight (TOF), TR/TE 50/4.2 msec, flip angle $45^{\circ}$, FOV $13 \mathrm{~cm}$, matrix size $256 \times 128$, and slice thickness 3.0 $\mathrm{mm}$. Three axial slices were selected (the most stenotic site, $3.0 \mathrm{~mm}$ proximal to the stenosis, and $3.0 \mathrm{~mm}$ distal to the stenosis), and the ratio of the mean signal intensity in the axial plaque area to the ipsilateral sternocleidomastoid muscle was quantified. ${ }^{9}$ Parameters of axial diffusion-weighted imaging were as follows: TR/TE 10,000/82 msec, 3 motion-probing gradient directions with a $b$ value of $1000 \mathrm{~s} / \mathrm{mm}^{2}$, matrix size $128 \times 192$, FOV $27 \mathrm{~cm}$, and slice thickness $5 \mathrm{~mm}$ with a 1-mm gap. The infarct volume was measured on all axial images using a manual tracing method and automatically calculated with Synapse software (version 3.2.1, Fujifilm Medical Systems).

\section{Intraoperative ICG Videoangiography}

The ICG videoangiography method was approved for clinical use by the ethics committee of Nagasaki University Hospital, and all patients gave written informed consent. ICG angiography was performed using the OPMI Pentero surgical microscope with integrated ICG technology (Carl Zeiss Co.). After exposing the carotid bifurcation, both intraarterial and intravenous ICG angiography examinations were separately performed to analyze the neovessel appearance (Fig. 1). For intraarterial ICG injection, a 21-gauge needle was introduced into the common carotid artery, and $0.05 \mathrm{mg}$ of ICG (Daiichi Pharmaceutical) in $2 \mathrm{ml}$ of normal saline was injected through the needle. With the intraarterial method, neovessels derived from the endothelial lumen were evaluated as NVe, which was defined as the early appearance and washout of the ICG within the endothelial neovessel. For intravenous ICG injection, $12.5 \mathrm{mg}$ of ICG in $5 \mathrm{ml}$ of normal saline was injected into the antecubital vein, and neovessels derived from the vasa vasorum on the adventitia were evaluated as NVv, which was defined as the delayed appearance of the ICG within the vasa vasorum 10 seconds after the appearance of the ICG in the carotid lumen. The appearance of no neovessels using both methods was categorized as "none." Intraarterial ICG allows for easier evaluation of $\mathrm{NVe}$ than $\mathrm{NVv}$ because of the high concentration and early washout of ICG. Conversely, intravenous ICG allows for easier evaluation of $\mathrm{NVv}$ because systemic (intravenous) ICG injection enables longer evaluation of the vessels.

\section{Pathological Procedure}

Whole specimens were formalin fixed, sectioned in 3.0-mm transverse slices, decalcified, and embedded in paraffin. Next, 5.0- $\mu \mathrm{m}$ sections, including the most stenotic slice, were subjected to histopathological analysis of the plaque phenotype after staining with $\mathrm{H} \& \mathrm{E}$, Azan, Berlin blue, and CD31. The areas of plaques showing IPH, hemosiderin deposits, neovessels, or inflammation were separately evaluated in the axial slices. The total IPH areas were measured on sections stained with $\mathrm{H} \& \mathrm{E}$ and Azan. For observation of the details of the luminal side of the plaque, the areas exhibiting hemosiderin deposits, neovessels, and inflammation were evaluated with Berlin blue, CD31, and H \& E, respectively. Inflammatory cells 

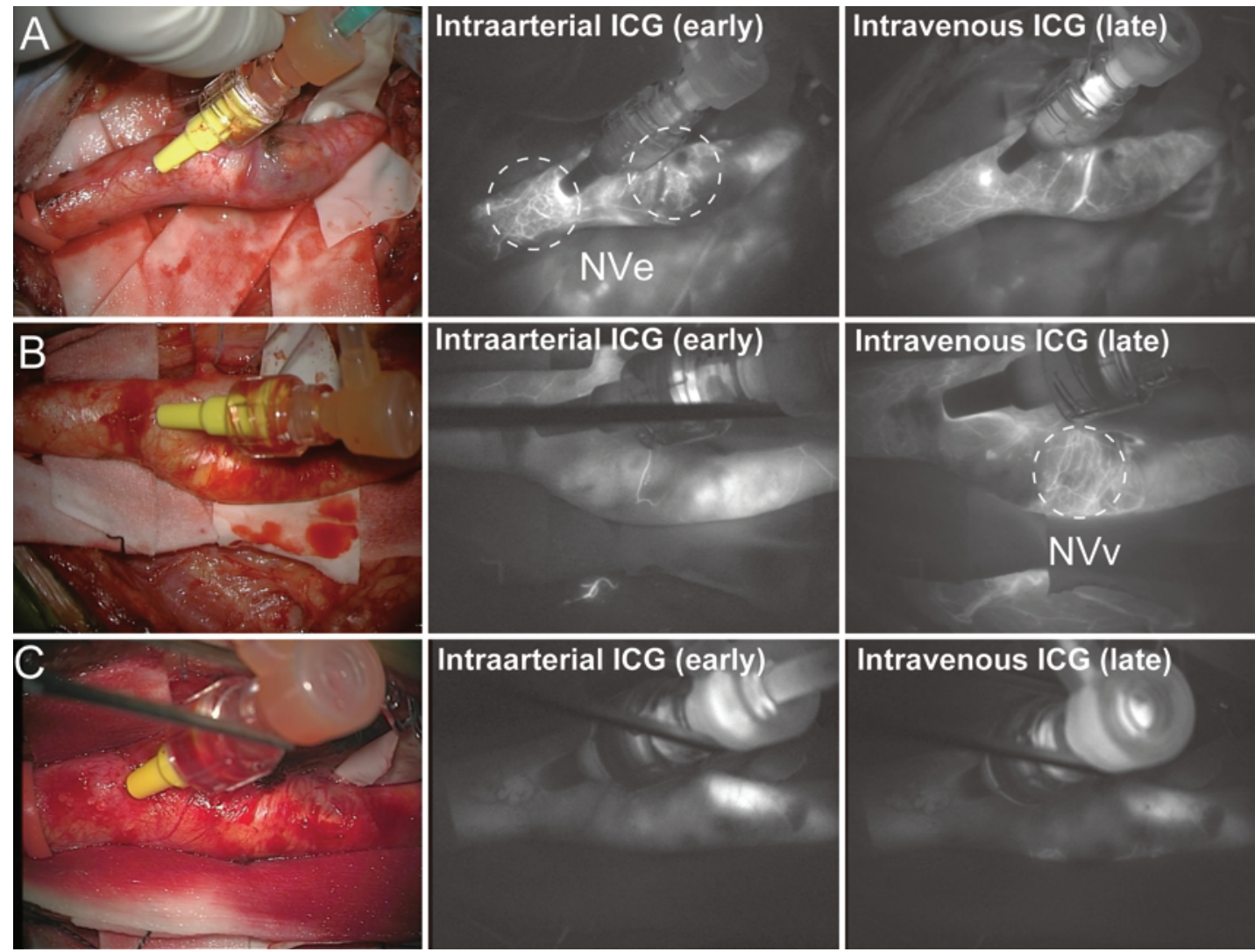

FIG. 1. The ICG-based neovessel appearance was categorized into 3 groups. A: Neovessels from the endothelium (NVe) were characterized by an immediate appearance and early washout of the ICG within the neovessels after intraarterial injection. B: Neovessels from the vasa vasorum (NVv) were characterized by a delayed appearance of the ICG within the vessels 10 seconds after the appearance of ICG in the carotid lumen with intravenous injection. C: Plaques showing no neovessels with both intraarterial and intravenous ICG injection were categorized as "none." The dashed circles show neovessels.

were identified by the presence of dark nuclear staining of macrophage-like cells observed with H \& E. Quantification of each area was performed using ImageJ software (version $1.45 \mathrm{~s}, \mathrm{NIH})$.

\section{Imaging, Pathological Review, and Data Analysis}

The ICG and MR images were separately analyzed by 2 experienced readers (N.H. and M.M.), and decisions were made by consensus. Both imaging readers were blinded to the radiological, histopathological, and clinical characteristics. The pathological slides were also blindly evaluated by 1 experienced reader (N.H.). To establish the reproducibility of our qualitative assessment, 2 different readers (N.H. and Y.M.) and 1 reader (N.H.) determined the interobserver and intraobserver agreement, respectively, with application of Cohen's $\kappa$ by grading the ICG findings at an interval of more than 7 days. Cohen's $\kappa$ was evaluated using an established grading of agreement: 0.00 (no agreement), 0.00-0.20 (poor), 0.21-0.40 (fair), 0.410.60 (moderate), $0.61-0.80$ (substantial), and $0.81-1.00$ (nearly perfect). Correlations between the TOF MRI findings and pathological tissue areas, or between pathological tissue areas, were analyzed by Pearson's rank correlation test, which measures the linear relationship between
2 variables, because we expected a linear relationship. Very weak, weak, moderate, strong, and very strong correlations were defined as $0.00-0.19,0.20-0.39,0.40-0.59$, $0.60-0.79$, and $0.80-1.00$, respectively.

\section{Statistical Analysis}

Data were tested for normality of distribution and equal standard deviations using GraphPad InStat (version 3.10, GraphPad Software) to determine whether parametric or nonparametric assumptions should be used for each statistical method. Comparisons between groups were performed using the Kruskal-Wallis test with Dunn's post hoc test for continuous variables and the chi-square test for categorical variables. Unless stated otherwise, differences were considered statistically significant at $\mathrm{p}<0.05$.

\section{Results}

\section{Patient Characteristics}

The characteristics of the study population are listed in Table 1. All patients who exhibited NVe also exhibited $\mathrm{NVv}$ and were included in the NVe group. Of the $57 \mathrm{pa}-$ tients, 13 exhibited NVe, 33 exhibited NVv, and 11 exhibited no neovessels based on intraoperative ICG video- 
TABLE 1. Characteristics of the study population

\begin{tabular}{|c|c|c|c|c|}
\hline \multirow[b]{2}{*}{ Variable } & \multicolumn{3}{|c|}{ ICG } & \multirow[b]{2}{*}{$p$ Value } \\
\hline & $\mathrm{NVe}(\mathrm{n}=13)$ & $\mathrm{NVv}(\mathrm{n}=33)$ & None $(n=11)$ & \\
\hline \multicolumn{5}{|l|}{ Cardiovascular risk factor } \\
\hline Mean age \pm SD (yrs) & $70.5 \pm 6.6$ & $71.9 \pm 7.8$ & $67.5 \pm 9.2$ & 0.28 \\
\hline Male sex (\%) & $13(100)$ & $27(81.8)$ & $11(100)$ & 0.09 \\
\hline Mean BMI $\pm \mathrm{SD}\left(\mathrm{kg} / \mathrm{m}^{2}\right)$ & $22.9 \pm 2.2$ & $23.7 \pm 3.0$ & $24.5 \pm 2.4$ & 0.38 \\
\hline Diabetes mellitus (\%) & $4(30.8)$ & $12(36.4)$ & $4(36.4)$ & 0.93 \\
\hline Hypertension (\%) & $10(76.9)$ & $27(81.8)$ & $11(100)$ & 0.26 \\
\hline Current or former smoker (\%) & $9(69.2)$ & $19(57.6)$ & $10(90.9)$ & 0.13 \\
\hline \multicolumn{5}{|l|}{ Use of drugs (\%) } \\
\hline Diabetes medication & $2(15.4)$ & $9(27.3)$ & $2(18.2)$ & 0.64 \\
\hline Antihypertensives & $8(61.5)$ & $26(78.8)$ & $10(90.9)$ & 0.23 \\
\hline Statins & $10(76.9)$ & $23(69.7)$ & $4(36.4)$ & 0.08 \\
\hline Antithrombotics & $13(100)$ & $33(100)$ & $11(100)$ & 1.00 \\
\hline Mean C-reactive protein \pm SD & $0.22 \pm 0.08$ & $0.16 \pm 0.05$ & $0.11 \pm 0.03$ & 0.32 \\
\hline \multicolumn{5}{|l|}{ Lipids (mean \pm SD) } \\
\hline LDL (mg/dl) & $100.5 \pm 35.7$ & $98.5 \pm 31.6$ & $100.4 \pm 25.1$ & 0.97 \\
\hline $\mathrm{HDL}(\mathrm{mg} / \mathrm{dl})$ & $42.5 \pm 14.8$ & $49.7 \pm 19.1$ & $39.3 \pm 7.5$ & 0.47 \\
\hline Total cholesterol (mg/dl) & $168.1 \pm 35.6$ & $175.5 \pm 45.1$ & $180.2 \pm 37.0$ & 0.77 \\
\hline Triglycerides (mg/dl) & $131.9 \pm 40.7$ & $118.9 \pm 63.7$ & $145.2 \pm 97.2$ & 0.52 \\
\hline \multicolumn{5}{|l|}{ Radiological findings } \\
\hline Mean degree of stenosis $\pm \mathrm{SD}\left({ }^{\circ}\right)$ & $83.0 \pm 11.3$ & $78.6 \pm 14.3$ & $82.7 \pm 10.1$ & 0.47 \\
\hline Ulceration (\%) & $2(15.4)$ & $10(30.3)$ & $0(0)$ & 0.09 \\
\hline Mean T1-weighted ratio \pm SD & $1.68 \pm 0.5$ & $1.56 \pm 0.5$ & $1.35 \pm 0.5$ & 0.21 \\
\hline Mean TOF ratio \pm SD & $1.71 \pm 0.6$ & $1.46 \pm 0.6$ & $1.36 \pm 0.6$ & 0.29 \\
\hline \multicolumn{5}{|l|}{ Clinical presentation (\%) } \\
\hline Embolic infarction (artery to artery) & $8(61.5)$ & $16(48.5)$ & $5(45.5)$ & 0.68 \\
\hline Transient ischemic attack & $3(23.1)$ & $1(3.0)$ & $1(9.1)$ & 0.10 \\
\hline Asymptomatic & $2(15.4)$ & $16(48.5)$ & $5(45.5)$ & 0.11 \\
\hline Mean days (diagnosis to surgery) \pm SD & $40.8 \pm 8.2$ & $36.2 \pm 9.5$ & $41.0 \pm 8.1$ & 0.16 \\
\hline
\end{tabular}

$\mathrm{BMI}=$ body mass index; $\mathrm{HDL}=$ high-density lipoprotein; $\mathrm{LDL}=$ low-density lipoprotein.

angiography. Overall, the interobserver and intraobserver reproducibilities of neovessel appearance were substantial for ICG $(\kappa=0.76)$ and at 7 days postoperatively $(\kappa=0.76)$. All patients routinely received antithrombotic agents (aspirin, clopidogrel, or both) after the diagnosis of carotid stenosis and continued these agents as perioperative management. For patients who received both agents, only aspirin was continued from 7 days preoperatively. There were no significant differences among the groups in terms of cardiovascular risk factors, drug use, C-reactive protein levels, lipid parameters, radiological findings, or clinical presentations, with the exception of a higher percentage of symptomatic presentations in the NVe group (artery-toartery embolic infarction in $61.5 \%$ and transient ischemic attack in $23.1 \%$ ). The interval from diagnosis to surgery ranged from 28 to 54 days (median 39 days) in symptomatic patients and from 18 to 56 days (median 37 days) in asymptomatic patients. There was no significant difference in this interval among the groups. Interestingly, patients with NVe had significantly larger infarctions on diffusion-weighted MRI than did patients with NVv (9675.0 $\pm 5601.9 \mathrm{~mm}^{3}$ vs $2306.6 \pm 856.9 \mathrm{~mm}^{3}$, respectively; $\mathrm{p}=$ 0.04; Fig. 2).

\section{Characteristics of Plaque Components With Different Neovessel Appearances}

There was a significant difference in plaque components among the various neovessel appearances (Fig. 3). Patients with NVe exhibited larger areas of IPH (47.2 \pm $\left.8.3 \mathrm{~mm}^{2}\right)$ than did patients with $\mathrm{NVv}\left(19.8 \pm 5.2 \mathrm{~mm}^{2}, \mathrm{p}<\right.$ $0.01)$ and patients without neovessels $\left(7.4 \pm 3.6 \mathrm{~mm}^{2}, \mathrm{p}<\right.$ 0.01 ; Fig. 3A). Moreover, the hemosiderin deposits around the lumen were larger in patients with $\mathrm{NVe}(0.5 \pm 0.2$ $\left.\mathrm{mm}^{2}\right)$ than in patients with $\mathrm{NVv}\left(0.2 \pm 0.1 \mathrm{~mm}^{2}, \mathrm{p}<0.05\right.$; Fig. 3B). The areas of neovessels or inflammation around the lumen were also larger in patients with NVe, but the difference did not reach statistical significance $(0.4 \pm 0.7$ vs $0.1 \pm 0.4 \mathrm{~mm}^{2}, \mathrm{p}=0.11$, and $1.0 \pm 1.1$ vs $0.6 \pm 0.9 \mathrm{~mm}^{2}$, $\mathrm{p}=0.26$, respectively; Fig. $3 \mathrm{C}$ and $\mathrm{D})$. MR plaque imaging showed a strong correlation between plaque intensity on TOF MRI and IPH $(r=0.61, p<0.0001)$, indicating that TOF MRI findings can serve as markers of IPH (data 


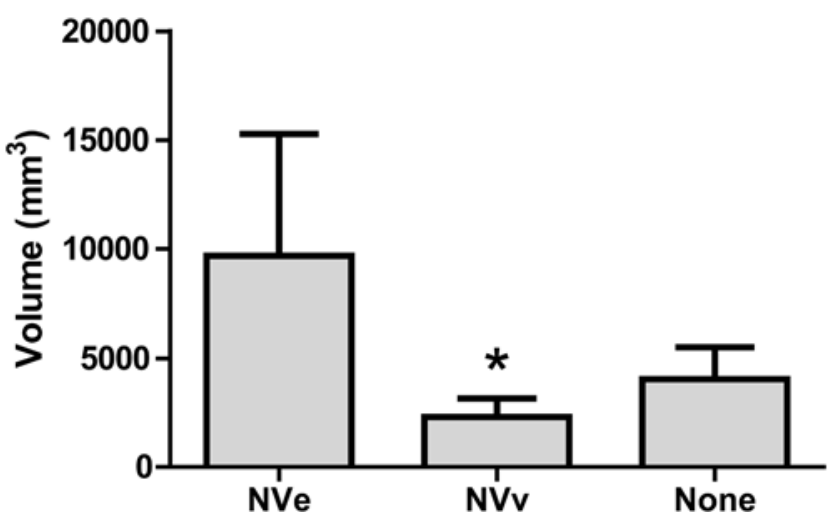

FIG. 2. Graph showing the infarction volume with diffusion-weighted MRI in the 3 groups. ${ }^{*} p<0.05$ compared with NVe, Mann-Whitney test, 2-tailed $p$ value.

not shown). Additionally, a moderate correlation was observed between TOF and neovessels $(r=0.44, p=0.001)$ and a weak correlation was observed between TOF and inflammation $(r=0.32, p=0.02)$.

\section{Correlation Between Inflammation and IPH}

Finally, we analyzed the role of inflammation in the development of IPH. There was a moderate correlation between inflammation and neovessels $(r=0.43, p=0.0008$; Fig. 4A) and a strong correlation between inflammation and hemosiderin spots $(\mathrm{r}=0.62, \mathrm{p}<0.0001$; Fig. 4B). These findings indicate the presence of an interaction between inflammation and neovessel formation leading to vessel integrity and old hemorrhage (hemosiderin) around the lumen, which could contribute to vessel vulnerability and IPH. Inflammation $(r=0.349, p=0.0097)$ or the neovessels themselves $(r=0.30, p=0.02)$ were also correlated with IPH, but the correlation was weak (Fig. 4C and D). This finding is similar to that of the TOF MRI analysis. These results indicate that inflammation and neovessel formation play a synergistic and important role in vessel breakdown. Representative cases are shown in Fig. 5.

\section{Discussion}

Neovascularization has been proposed as one of the critical factors of plaque vulnerability in patients with carotid stenosis and may contribute to the development of IPH. , $15,17,25,26$ Initially, adventitial enhancement was proposed as a marker of neovascularization with ultrasound, CT angiography, and MRI, ${ }^{1,712,23}$ and the vasa vasorum on the adventitia is believed to be an appropriate target. However, progression of the adventitial vasa vasorum into the intima is reportedly a critical factor for both IPH development and plaque enlargement according to histological analysis; this has not yet been a focus of imaging analysis. ${ }^{1,6,15}$ Some reports have alternatively evaluated contrast enhancement of the plaque itself with various imaging modalities, and contrast enhancement is a reported marker of IPH. ${ }^{9-10,16}$ Nevertheless, some authors argue that neovascularization alone cannot be the only factor involved
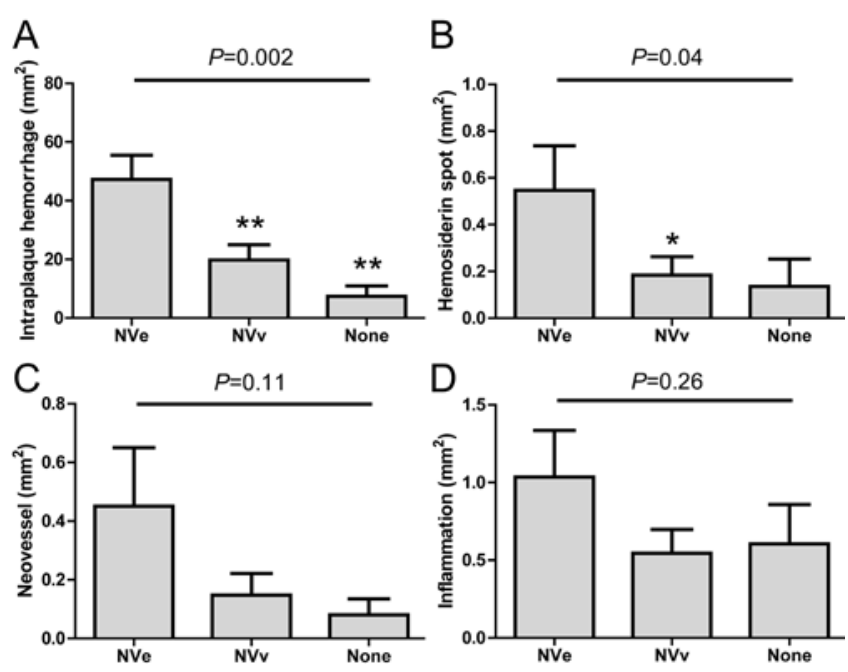

FIG. 3. Graphs showing the areas of IPH (A), hemosiderin spots (B), neovessels (C), and inflammation (D) based on specific staining in the 3 groups. ${ }^{*} p<0.05,{ }^{* *} p<0.01$ compared with NVe. Kruskal-Wallis test with Dunn's post hoc test.

in plaque enhancement because some plaques with high enhancement do not show neovascularization, and the pathophysiological and clinical significance of plaque enhancement remain undetermined. ${ }^{20,21}$ Therefore, dynamic assessment of neovessels has great clinical significance because it may confirm the relationship between advancement of the neovessels and the patient's clinical presentation.

In this study, we performed dynamic assessment of neovascularization with ICG videoangiography and provided evidence that communication of inwardly projecting neovessels with the lumen is associated with a high rate of symptomatic presentations and with a larger stroke
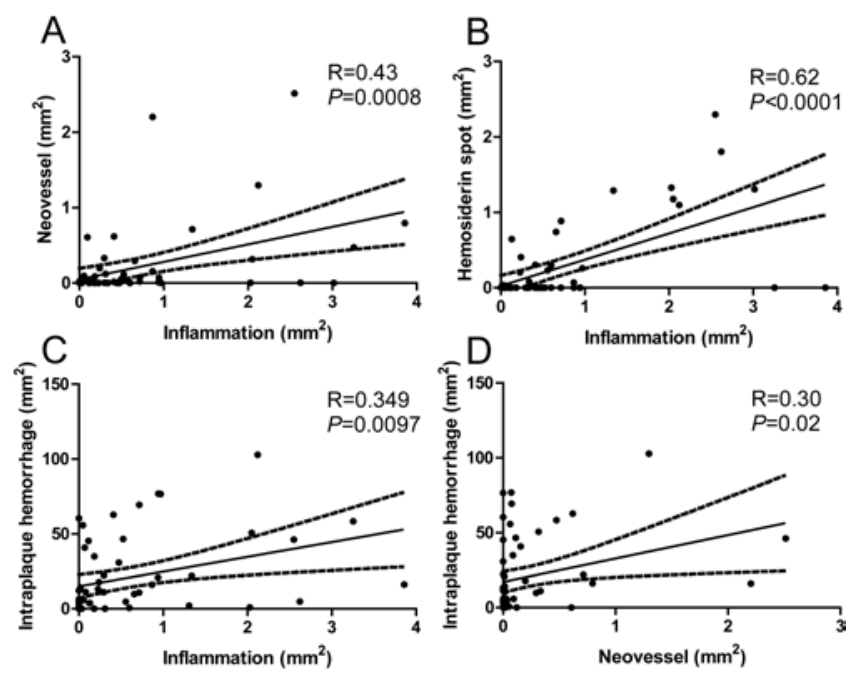

FIG. 4. Graphs showing correlations between inflammation and each plaque component around the lumen: neovessels (A), hemosiderin spots $(\mathrm{B})$, and IPH (C). Note that inflammation or the neovessels themselves were also correlated with $\mathrm{IPH}$, but the correlation was weak (C and $\mathrm{D}$ ). 
A
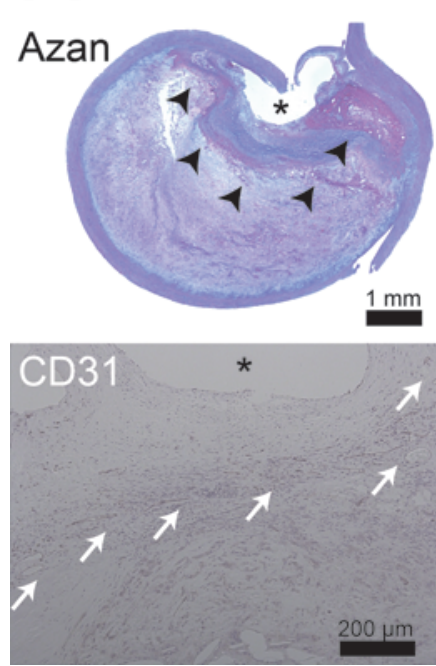
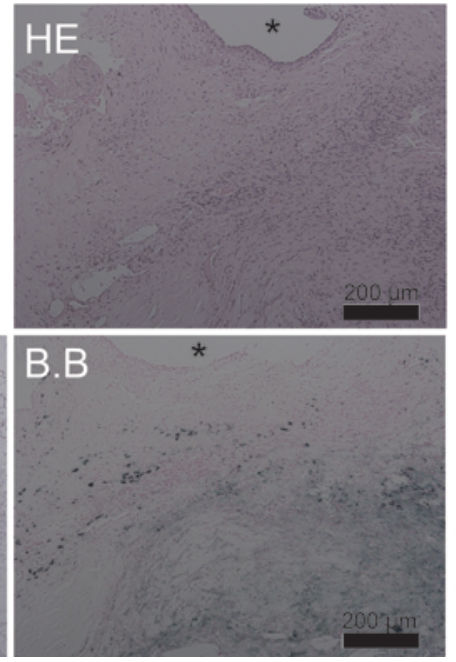

B
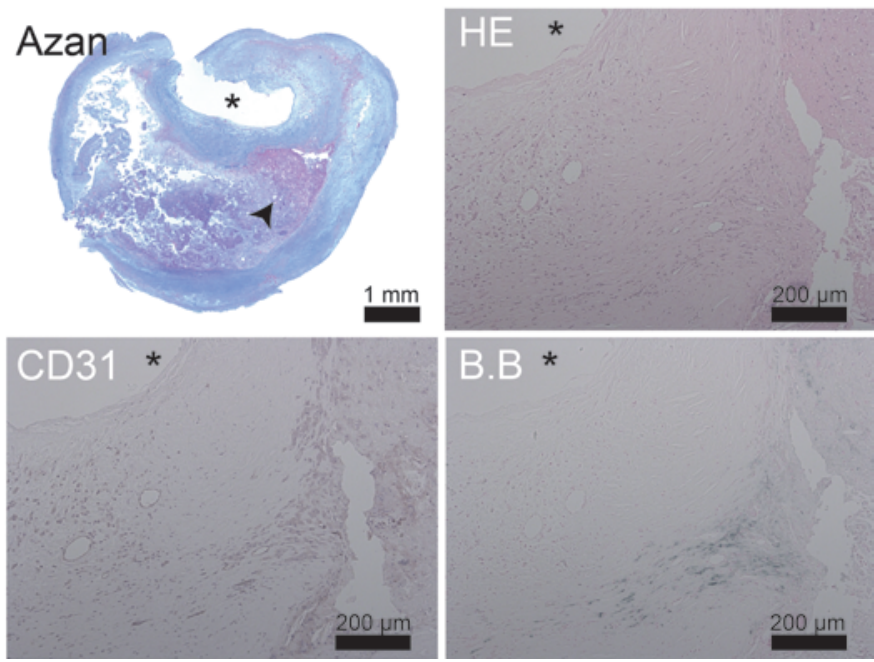

FIG. 5. Representative cases of NVe (A) and NVv (B). Azan staining shows much more severe IPH in NVe around the fibrous cap than in NVv (arrowheads, A and B). H \& E (HE) staining shows more inflammatory cells and CD31 staining shows more advanced formation of tiny, deformed neovessels around the fibrous cap (arrows, A) in NVe than in NVv. Hemosiderin spots were also much more severe in NVe than in NVv. Asterisk = lumen; B.B = Berlin blue.

size following artery-to-artery embolism. Contrast-enhanced ultrasound imaging has provided similar findings, as contrast media has been observed to flow from the lumen into the plaque, ${ }^{23}$ supporting our findings. Moreover, we showed that the inflammatory response around the lumen was correlated with these neovessels, which produce continuing minor, chronic hemorrhage near the lumen. We believe that such luminal (endothelial)-connected inwardly projecting neovessels are more specific for IPH than is the vasa vasorum. Plaque inflammation was previously reported to be associated with the highest risk of stroke recurrence ${ }^{14}$ but the interaction between neovascularization and inflammation remains undetermined. Truijman et al. ${ }^{25}$ reported that ${ }^{18}$ fluorine-fluorodeoxyglucose uptake is weakly associated with increased vasa vasorum on the plaque and concluded that inflammation- and vasa vasorum-based neovascularization are not interchangeable.
This also supports our hypothesis that vasa vasorum-focused neovascularization is not specific for IPH and that inwardly projecting neovascularization and inflammation may synergistically contribute to IPH. If this hypothesis is fully established, a treatment strategy focused on antiinflammation might be beneficial with respect to prevention of IPH.

Generally, neovascularization is believed to involve the growth and extension of adventitial blood vessels (vasa vasorum) into the intima in response to tissue hypoxia, and this occurs when the intima thickens beyond the diffusion limits of oxygen and nutrients. ${ }^{1,6}$ Increased levels of hypoxia-inducible factor $\alpha$, a transcription factor that is upregulated under hypoxic conditions and that promotes hypoxia-dependent neovascularization, have been found in human atherosclerotic plaques. ${ }^{22}$ Michel et al. ${ }^{15}$ recently provided new evidence supporting the progres-
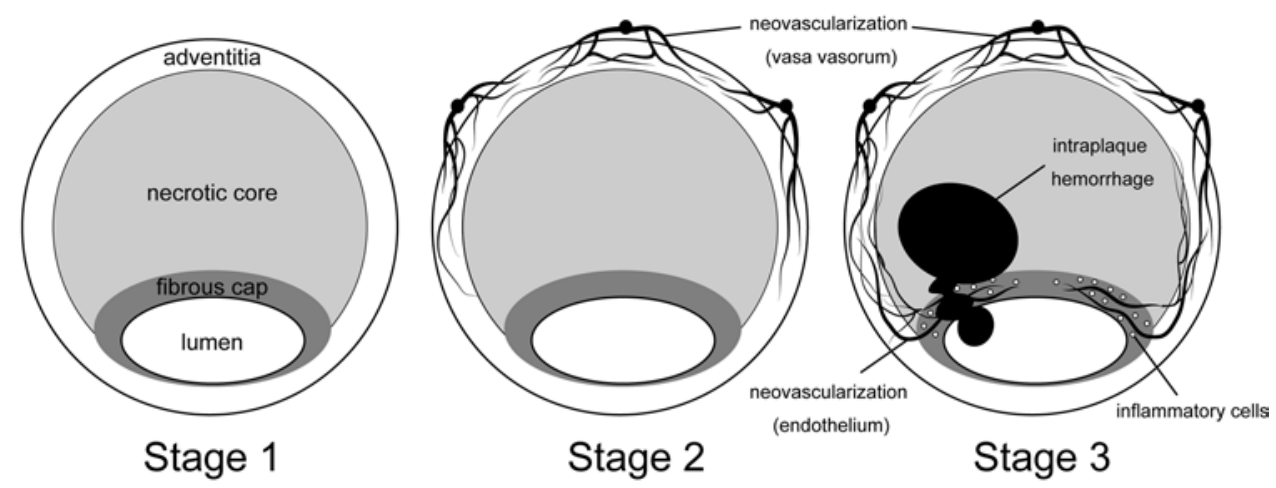

FIG. 6. Schema showing advancement of neovascularization in a carotid plaque. Stage 1: No development of neovessels. Stage 2: A developed vasa vasorum is present, but it is located only in the adventitia. Stage 3: Neovessels are sprouting into the luminal side in association with inflammatory cells, which communicate with the lumen. 
sion of neovascularization from the adventitia toward the plaque across the media, initiation of neovascularization by lipid mediators generated by the plaque on the luminal side, and outward connection to the medial smooth muscle cells. These observations were based on pathological analysis, and we further confirmed that luminal neovessel sprouting and communication with the lumen is correlated with artery-to-artery embolic stroke under conditions of inflammation. Nevertheless, how luminal neovessels communicate with the vessel lumen remains unclear. One pathological analysis showed the orifice of the neovessels on the luminal surface. ${ }^{13}$ Conversely, another pathological analysis showed that the luminal interface contained yellow fatty streaks and tissue blood deposits without orifices of the neovessels. ${ }^{15}$ These supportive findings indicate that direct or indirect communication between the lumen and neovessels could contribute to IPH accompanied by an inflammatory response. Taken together, neovessel sprouting with inflammation and connection with the lumen could represent an advanced stage of neovascularization associated with plaque vulnerability and IPH (Fig. 6).

Several limitations of this study should be addressed. First, analysis of the neovessel pattern based on ICG videoangiography was not quantitative, and the results may have been affected by the plaque thickness and calcified adventitia in some cases. Second, the number of patients in this study was relatively small, making it difficult to obtain substantial power for our hypothesis, although different characteristics were observed depending on the appearance of the neovessels with dynamic assessment using ICG. Third, although cerebral events distal to an atherosclerotic carotid stenosis are believed to be mostly embolic and to originate from the unstable plaque, they may also be due to hemodynamic impairment in cases of severe carotid stenosis. ${ }^{24}$ This study may have included some patients with symptomatic carotid stenosis mainly due to hemodynamic impairment that could not be clearly identified, although there is no evidence regarding the degree to which hemodynamic impairment contributes to cerebral infarction. Further radiological studies are required to establish the correlation between the inwardly projecting neovessel appearance and a symptomatic presentation. Nevertheless, this condition could be a new therapeutic target, and regression of luminal neovessel sprouting and inflammation may help to prevent IPH development and a symptomatic presentation.

\section{Conclusions}

This study provides evidence that communication of inwardly projecting neovessels with the lumen and inflammation are synergistically associated with a high risk of a symptomatic presentation via IPH beyond progression of the atheroma.

\section{References}

1. Barger AC, Beeuwkes R III, Lainey LL, Silverman KJ: Hypothesis: vasa vasorum and neovascularization of human coronary arteries. A possible role in the pathophysiology of atherosclerosis. N Engl J Med 310:175-177, 1984

2. Chalela JA: Evaluating the carotid plaque: going beyond stenosis. Cerebrovasc Dis 27 (Suppl 1):19-24, 2009
3. Cicha I, Wörner A, Urschel K, Beronov K, Goppelt-Struebe $\mathrm{M}$, Verhoeven E, et al: Carotid plaque vulnerability: a positive feedback between hemodynamic and biochemical mechanisms. Stroke 42:3502-3510, 2011

4. Doyle B, Caplice N: Plaque neovascularization and antiangiogenic therapy for atherosclerosis. J Am Coll Cardiol 49:2073-2080, 2007

5. European Carotid Surgery Trialists' Collaborative Group: Randomised trial of endarterectomy for recently symptomatic carotid stenosis: final results of the MRC European Carotid Surgery Trial (ECST). Lancet 351:1379-1387, 1998

6. Finn AV, Jain RK: Coronary plaque neovascularization and hemorrhage: a potential target for plaque stabilization? JACC Cardiovasc Imaging 3:41-44, 2010

7. Granada JF, Feinstein SB: Imaging of the vasa vasorum. Nat Clin Pract Cardiovasc Med 5 (Suppl 2):S18-S25, 2008

8. Hoogi A, Adam D, Hoffman A, Kerner H, Reisner S, Gaitini D: Carotid plaque vulnerability: quantification of neovascularization on contrast-enhanced ultrasound with histopathologic correlation. AJR Am J Roentgenol 196:431-436, 2011

9. Horie N, Morikawa M, Ishizaka S, Takeshita T, So G, Hayashi K, et al: Assessment of carotid plaque stability based on the dynamic enhancement pattern in plaque components with multidetector CT angiography. Stroke 43:393-398, 2012

10. Kawahara I, Morikawa M, Honda M, Kitagawa N, Tsutsumi $\mathrm{K}$, Nagata I, et al: High-resolution magnetic resonance imaging using gadolinium-based contrast agent for atherosclerotic carotid plaque. Surg Neurol 68:60-66, 2007

11. Kernan WN, Ovbiagele B, Black HR, Bravata DM, Chimowitz MI, Ezekowitz MD, et al: Guidelines for the prevention of stroke in patients with stroke and transient ischemic attack: a guideline for healthcare professionals from the American Heart Association/American Stroke Association. Stroke 45:2160-2236, 2014

12. Kerwin WS, Oikawa M, Yuan C, Jarvik GP, Hatsukami TS: MR imaging of adventitial vasa vasorum in carotid atherosclerosis. Magn Reson Med 59:507-514, 2008

13. Kumamoto M, Nakashima Y, Sueishi K: Intimal neovascularization in human coronary atherosclerosis: its origin and pathophysiological significance. Hum Pathol 26:450-456, 1995

14. Marnane M, Prendeville S, McDonnell C, Noone I, Barry M, Crowe M, et al: Plaque inflammation and unstable morphology are associated with early stroke recurrence in symptomatic carotid stenosis. Stroke 45:801-806, 2014

15. Michel JB, Martin-Ventura JL, Nicoletti A, Ho-Tin-Noé B: Pathology of human plaque vulnerability: mechanisms and consequences of intraplaque haemorrhages. Atherosclerosis 234:311-319, 2014

16. Millon A, Boussel L, Brevet M, Mathevet JL, Canet-Soulas E, Mory C, et al: Clinical and histological significance of gadolinium enhancement in carotid atherosclerotic plaque. Stroke 43:3023-3028, 2012

17. Moreno PR, Purushothaman KR, Sirol M, Levy AP, Fuster $\mathrm{V}$ : Neovascularization in human atherosclerosis. Circulation 113:2245-2252, 2006

18. Nakaoka T, Shoji H, Tabeta H, Ikeshima H, Uchida T, Itou $\mathrm{K}$ : [Carotid artery wall perfusion of the plaque: three dimensional contrast sonographic imaging.] No Shinkei Geka 36:991-1000, 2008 (Jpn)

19. North American Symptomatic Carotid Endarterectomy Trial Collaborators: Beneficial effect of carotid endarterectomy in symptomatic patients with high-grade carotid stenosis. $\mathbf{N}$ Engl J Med 325:445-453, 1991

20. Saba L, Lai ML, Montisci R, Tamponi E, Sanfilippo R, Faa $\mathrm{G}$, et al: Association between carotid plaque enhancement shown by multidetector CT angiography and histologically validated microvessel density. Eur Radiol 22:2237-2245, 2012 
21. Saba L, Tamponi E, Raz E, Lai L, Montisci R, Piga M, et al: Correlation between fissured fibrous cap and contrast enhancement: preliminary results with the use of CTA and histologic validation. AJNR Am J Neuroradiol 35:754-759, 2014

22. Sluimer JC, Gasc JM, van Wanroij JL, Kisters N, Groeneweg M, Sollewijn Gelpke MD, et al: Hypoxia, hypoxia-inducible transcription factor, and macrophages in human atherosclerotic plaques are correlated with intraplaque angiogenesis. J Am Coll Cardiol 51:1258-1265, 2008

23. Staub D, Patel MB, Tibrewala A, Ludden D, Johnson M, Espinosa $\mathrm{P}$, et al: Vasa vasorum and plaque neovascularization on contrast-enhanced carotid ultrasound imaging correlates with cardiovascular disease and past cardiovascular events. Stroke 41:41-47, 2010

24. Szabo K, Kern R, Hennerici MG: Recent advances in imaging in management of symptomatic internal carotid artery disease. Int J Stroke 2:97-103, 2007

25. Truijman MT, Kwee RM, van Hoof RH, Hermeling E, van Oostenbrugge RJ, Mess WH, et al: Combined 18F-FDG PET$\mathrm{CT}$ and DCE-MRI to assess inflammation and microvascularization in atherosclerotic plaques. Stroke 44:3568-3570, 2013
26. Yoshimura S, Yamada K, Kawasaki M, Asano T, Kanematsu M, Takamatsu M, et al: High-intensity signal on time-offlight magnetic resonance angiography indicates carotid plaques at high risk for cerebral embolism during stenting. Stroke 42:3132-3137, 2011

\section{Author Contributions}

Conception and design: Horie. Acquisition of data: Horie, Tsujino. Analysis and interpretation of data: Horie, Morofuji, Morikawa, Tateishi, Izumo. Drafting the article: Horie. Critically revising the article: Horie. Reviewed submitted version of manuscript: Horie. Approved the final version of the manuscript on behalf of all authors: Horie. Statistical analysis: Horie. Administrative/technical/material support: Horie. Study supervision: Horie, Hayashi, Nagata.

\section{Correspondence}

Nobutaka Horie, Department of Neurosurgery, Nagasaki University School of Medicine, 1-7-1 Sakamoto, Nagasaki 8528501, Japan.email: nobstanford@gmail.com. 